
\title{
$\begin{array}{ll}\text { Research Square } & \begin{array}{l}\text { Preprints are preliminary reports that have not undergone peer review. } \\ \text { They should not be considered conclusive, used to inform clinical practice, } \\ \text { or referenced by the media as validated information. }\end{array}\end{array}$
}

\section{The Efficacy and Safety of High-Dose Nonsedating Antihistamines in Chronic Spontaneous Urticaria: A Systematic Review of Randomized Clinical Trials}

\author{
Xianjun Xiao \\ Chengdu University of Traditional Chinese Medicine \\ Yunzhou Shi \\ Chengdu University of Traditional Chinese Medicine \\ Junpeng Yao \\ Chengdu University of Traditional Chinese Medicine \\ Wei Cao \\ Chengdu University of Traditional Chinese Medicine \\ Leixiao Zhang \\ West China Hospital of Sichuan University \\ Zihao Zou \\ Chengdu University of Traditional Chinese Medicine \\ Siyuan Zhou \\ Chengdu University of Traditional Chinese Medicine \\ Chuan Wang \\ Wuhan No.1 Hospital \\ Mingling Chen \\ Affiliated Hospital of Chengdu University of Traditional Chinese Medicine \\ Rongjiang Jin \\ Chengdu University of Traditional Chinese Medicine \\ Ying Li ( $\square$ liying@cdutcm.edu.cn ) \\ Chengdu University of Traditional Chinese Medicine \\ Qianhua Zheng \\ Chengdu University of Traditional Chinese Medicine
}

\section{Research Article}

Keywords: nonsedating antihistamines, chronic spontaneous urticaria, safety, high-dose, systematic review

Posted Date: January 20th, 2022

DOI: https://doi.org/10.21203/rs.3.rs-1070216/v1

License: @ (1) This work is licensed under a Creative Commons Attribution 4.0 International License. Read Full License 


\section{Abstract \\ Background}

Standard doses of second-generation $\mathrm{H}_{1}$-antihistamines ( $\mathrm{sgAHs}$ ) as first-line treatment are not always effective in treating chronic spontaneous urticaria (CSU), and hence an increase in the dose of sgAHs is recommended. However, literature evaluating the ef $\square$ cacy and safety of this treatment remains inconclusive, highlighting the need for a meta-analysis. The aim of this meta-analysis was to evaluate the ef $\square$ cacy and safety of high-dose sgAHs compared with standard-dose sgAHs in treating CSU.

\section{Methods}

A systematic literature search of double-blind, randomized controlled trials (RCT) utilizing multiple doses of sgAHs was performed by searching the electronic databases Medline, Embase, PsycINFO, Cochrane databases, and Web of Science. The response rate, the number of adverse events, somnolence, and withdrawal due to adverse events were extracted from each article. The data were combined and analyzed to quantify the safety and efficacy of the treatment.

\section{Results}

A total of 12 studies were identified, six of which met the eligibility criteria for the meta-analysis. Our pooled meta-analyses showed that high-dose sgAHs was associated with a significantly higher response rate than standard-dose (RR $1.12,95 \% \mathrm{Cl} 1.01$ to $1.24 ; \mathrm{P}=0.04$ ). Conversely, high doses of sgAHs were associated with significantly higher somnolence rates than standard dose (RD $0.05,95 \% \mathrm{Cl} 0.01$ to $0.09 ; \mathrm{P}=0.02)$. There was no significant difference in adverse events or withdrawal due to adverse events between standard- and high-dose treatments.

\section{Conclusions}

Our analyses showed that a high dose of sgAHs (up to two times the standard dose) might be more effective than a standard dose in CSU treatment. Highdose and standard-dose sgAHs showed similar adverse events, except for somnolence, where incidence was found to be dose-dependent in some studies. However, given the limited number of studies, our meta-analysis results should be interpreted with caution.

\section{Background}

Chronic spontaneous urticaria (CSU), also known as chronic idiopathic urticaria, is a condition characterized by the occurrence of spontaneous wheals, angioedema, or both for more than six weeks[1]. The prevalence of chronic urticaria around the world is estimated to be in the range of approximately $0.1-$ $1.4 \%$, and its prevalence appears to be increasing[2, 3]. CSU patients often experience numerous distressing symptoms, including sleep disturbances, fatigue, and psychological distress, leading to a profound reduction in their quality of life[3-6] and a substantial burden for health care systems[7-9].

CSU is typically managed using second-generation $\mathrm{H}_{1}$-antihistamines (sgAHs)[3]. The European[1] and American guidelines[10] recommend the use of sgAHs at licensed doses as the first-line treatment for CSU. In CSU patients with insufficiently controlled symptoms, guidelines[1, 10] recommend increasing the dose of sgAHs as a second-line treatment. Most studies on CSU reported on the safety and efficacy of standard-dose sgAHs, while studies evaluating the impact of high-dose sgAHs are mostly small and with low quality. Therefore, evidence for the high-dose of sgAHs in CSU is still limited. One straightforward approach to overcome the limitations of current studies is to combine available data through a meta-analysis[11]. Guillén-Aguinaga et al.[12]presented a meta-analysis with a focus on sgAHs dosing for CSU. The study found that updosing sgAHs signilcantly improved control of pruritus but not the number of wheals.

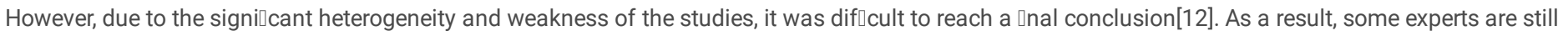
concerned that updosing sgAHs might increase adverse events[13,14]. Furthermore, the study by Guillén-Aguinaga et al.[12] did not evaluate the safety of using high-dose sgAHs, highlighting the need of a meta-analysis. We therefore performed a meta-analysis of randomized controlled trials (RCT) to evaluate the efficacy and side effects of high-dose sgAHs in the treatment of CSU, in order to provide new evidence for its clinical application.

\section{Methods}

The protocol for this review study was registered in the International Prospective Register of Systematic Reviews (PROSPERO) as CRD42020195864 and followed the Preferred Reporting Items for Systematic Reviews and Meta-analysis recommendations[15]. A systematic literature search was performed using the electronic databases PubMed, Scopus, Cochrane databases, and Web of Science. The search strategy included all published articles up to March 2020 and utilized the keywords "randomized controlled trials", "urticarial", "hives", "h1 antihistamine", and "second generation h1 Antihistamine". Furthermore, the bibliographies of any identified RCT and review articles were also analyzed to identify additional published or unpublished data.

\section{Eligibility Criteria For Systematic Review}

To be eligible for systematic review: (1) All double-blind RCT of patients with CSU that compared two or more fixed-doses sgAHs in their treatment groups (i.e. an active drug with placebo, or two or more doses of an active drug with or without placebo), (2) the study reported any of the following outcomes: the response rates (defined as pruritus symptoms reduction higher than 50\%, or at least a moderate to very good global symptom improvement. ); MPS (mean pruritus score, reflecting the overall situation of pruritus); MNW (mean number of wheals, reflecting the overall situation of wheals); MTSS (calculated as the 
sum of MPS and MNW, reflecting the overall situation of urticaria symptoms), DLQI (dermatology life quality index) and/or adverse events of the treatment; moreover, the eligible studies did not need to report all of the aforementioned outcomes but had to report response rates or adverse events, (3) Only articles published in English were included.

Studies were excluded if the full-text was inaccessible or if they had insufficient data for data pooling and analysis. All case reports, case series, review articles, in vitro studies, comments, and replies were also excluded.

\section{Study Selection}

The search result was evaluated by two independent reviewers (YZS and LXZ), and any disagreements were resolved by discussion with a third reviewer (XJX).

\section{Data Extraction And Bias Assessments}

The following information was independently extracted from the full text by two reviewers (WC and RJ): first author, year of publication, sex of patients, the number of sgAHs-treated patients, dose and treatment duration of sgAHs, outcome measurements, treatment response rates and change in CSU activity after treatment with sgAHs, as well as numbers of adverse events.

The quality and risk of bias of the included RCT studies were assessed using the Cochrane collaboration tool[16]. If the data in the study were incomplete, the original author was first contacted to obtain the corresponding data. Alternatively, the change in the response rate score after treatment from baseline was calculated using the formula recommended by the Cochrane handbook[16]. When the data were only presented in graphs, Adobe Photoshop (Adobe, Inc., San Jose, CA) was utilized to extract data[17, 18]. Studies were excluded if any of the above methods failed to provide sufficient data for analysis.

\section{Statistical analysis}

Data collection and analysis were performed using the RevMan V5.3 statistical software provided by the Cochrane collaboration. Random effects models were used for the meta-analysis because of the wide heterogeneity in the design, populations, and sample size between studies[19]. If the quantitative analysis was not appropriate, a descriptive analysis was provided. For continuous data, mean difference and $95 \%$ confidence interval (Cl) were used to measure the treatment effect. For dichotomous data, risk ratio (RR) with $95 \% \mathrm{Cl}$ were used to measure the treatment effect. In the case of studies with zero events in both arms, the risk difference (RD) was calculated[20]. The researcher agreement and a meta-analysis manual for the three-arm RCT of the Cochrane alliance were used to compare the outcomes in the two control groups [16].

\section{Results}

Through the literature search, a total of 3662 potentially eligible research articles were identified from the following databases (Figure 1): Medline ( $\mathrm{n}=488)$, Embase $(n=1086)$, Cochrane library $(n=765)$, PsycINFO $(n=34)$ and Web of Science $(n=1289)$. Six additional articles were identified by manually searching the reference lists of the articles of interest. Twelve RCTs, one triple-blind and eleven double-blind, were finally included in the meta-analysis[21-32].

Among the 2008 patients with CSU (Table. 1), sex data were reported by 10 of the studies (587 male and 1329 female patients) conducted in eight countries (Spain, the United States, Germany, United Kingdom, Japan, Colombia, Bulgaria, and France). Different types of sgAHs including fexofenadine, rupatadine, bilastine, desloratadine, cetirizine, levocetirizine, ebastine, and acrivastine were administered to $880,609,231,99,99,40,30$, and 20 patients with CSU, respectively. Fexofenadine was the most studied treatment (4/12 studies) $[21,23,28,29]$. The age of the patients ranged from 12 to 74 years. The evaluated studies analyzed different treatment outcomes. Four studies evaluated the response rates[21, 22, 27, 32], five studies evaluated adverse events[21-23, 27, 28] and four studies analyzed the somnolence[21, 22, 27, 32] and withdrawal of patients (due to adverse events) of sgAHs treatment[23, 27, 28]. The risk of bias of the included studies is presented in Figure 2.

\section{High-dose Versus Standard-dose Sgahs Response rate}

The response was reported in four studies (Figure 3A)[21, 22, 27, 32] with a total of 352 patients treated with high-dose and 310 patients treated with standarddose sgAHs. High-dose sgAHs was associated with a significantly higher response rate when compared with standard dose (RR $1.12,95 \% \mathrm{Cl} 1.01$ to 1.24 ; $\mathrm{P}=$ 0.04).

\section{Adverse events}

Adverse events (Figure 3B) were reported in five studies[21-23, 27, 28] with a total of 1053 patients evaluated (high dose $n=628 ;$ standard dose $n=425$ ). There was no significant difference in patients with CSU who experienced at least one adverse event between high-dose and standard-dose treatments (RR 1.05 , $95 \%$ Cl 0.92 to $1.21 ; P=0.44)$.

\section{Somnolence}


Somnolence (Figure 3C) was reported in four studies[21, 22, 27, 32] including 666 patients (high dose $n=354$; standard dose $n=312$ ). A high-dose sgAHs was associated with significantly higher somnolence rates when compared with the standard dose (RD $0.05,95 \% \mathrm{Cl} 0.01$ to 0.09 ; $\mathrm{P}=0.02)$. Due to the noted signi[cant heterogeneity between the included studies $\left(I^{2}=52 \%\right)$, the leave-one-out analysis was performed. When the study by Dubertret et al.[32] was excluded from the analysis, there was no significant difference in somnolence between high dose and standard dose (RD $0.02,95 \% \mathrm{Cl}-0.02$ to 0.06 ; $\mathrm{P}=0.36$ ).

\section{Withdrawal of patients due to adverse events}

Withdrawal of patients due to adverse events (Figure 3D) was reported in three studies[23, 27, 28] including 725 patients (high dose $n=442$; standard dose $\mathrm{n}=283$ ). There was no significant difference in the number of patients withdrawing from treatment due to adverse events between high-dose and standarddose treatments (RR $0.63,95 \% \mathrm{Cl} 0.29$ to $1.36 ; \mathrm{P}=0.24$ ).

\section{Assessment Of Treatment Efficacy Rupatadine}

Gimenez-Arnau et al.[22] reported that rupatadine provided over six weeks using doses of $10 \mathrm{mg}$ and $20 \mathrm{mg}$ led to a decrease in the mean pruritus score (MPS) from baseline by $59.5 \%$ and $66.1 \%$, respectively. Both doses resulted in a significantly improved MPS score but did not result in a significant reduction in the mean number of wheals (MNW) score when compared with the placebo. Furthermore, both doses of rupatadine effectively relieved the symptoms of CSU after the first administration. The MPS, MNW, and total symptom scores did not differ between doses at different time points. However, $10 \mathrm{mg}$ rupatadine had an overall better adverse event profile when compared with $20 \mathrm{mg}$ rupatadine.

Hide et al.[27] reported a mean total pruritus score (TPS) difference of -1.956 and -2.121 for $10 \mathrm{mg}$ and 20 mg rupatadine, compared with the placebo, respectively. There was no statistically significant difference between $10 \mathrm{mg}$ and $20 \mathrm{mg}$ rupatadine for TPS and MNW

Dubertret et al.[32] reported on patients with CSU treated with $5 \mathrm{mg}, 10 \mathrm{mg}$, and $20 \mathrm{mg}$ rupatadine once daily for four weeks. Over the four weeks treatment period, 10 and $20 \mathrm{mg}$ rupatadine resulted in a significant reduction in pruritus severity of $62.7 \%$ and $72.3 \%$, respectively, compared with $45.8 \%$ with placebo. Rupatadine at $5 \mathrm{mg}$ resulted in a reduction in the pruritus severity of $51.6 \%$. However, this reduction was not statistically significant when compared with the placebo. Rupatadine at $5 \mathrm{mg}, 10 \mathrm{mg}$, and $20 \mathrm{mg}$ led to a decrease in the MNW from baseline by $34.3 \%, 45.2 \%$, and $57.8 \%$, respectively over the four weeks treatment period, but this reduction was not statistically significant when compared with the placebo, which resulted in a reduction of $30.1 \%$.

\section{Fexofenadine}

Finn et al.[23] reported that bidaily (bid) doses of fexofenadine at $20 \mathrm{mg}, 60 \mathrm{mg}, 120 \mathrm{mg}$, and $240 \mathrm{mg}$, provided over four weeks, led to a significant reduction in pruritus severity and the number of wheals in CSU patients when compared with the placebo. Efficacy results in reducing pruritus were similar in the 60,120 , and $240 \mathrm{mg}$ groups and better when compared with the $20 \mathrm{mg}$ group. However, the 120 and $140 \mathrm{mg}$ groups resulted in a reduction in MNW and MTSS scores when compared with the $60 \mathrm{mg}$ group, although the difference was not statistically significant.

Nelson et al.[28] reported reductions in pruritus severity from baseline of $19 \%, 38 \%, 54 \%, 43 \%$, and $57 \%$ in the placebo, 20, 60,120 , and $240 \mathrm{mg}$ bid fexofenadine dose groups, respectively, as well as reductions in the MNW from baseline of $18 \%, 35 \%, 50 \%, 64 \%$, and $54 \%$ in the placebo $20,60,120$, and 240 $\mathrm{mg}$ bid fexofenadine dose groups, respectively. All fexofenadine $\mathrm{HCl}$ doses were statistically superior to placebo in reducing MPS and MNW scores but resulted in increased levels of interference with sleep and daily activities in a significant linear trend with dose.

Paul et al.[21] reported that approximately $73-81 \%$ of CSU patients receiving $60 \mathrm{mg}, 120 \mathrm{mg}, 180 \mathrm{mg}$, and $240 \mathrm{mg}$ of fexofenadine were considered to have a significant improvement in TSS compared with $54 \%$ in the placebo group, with the $120 \mathrm{mg}$ and $240 \mathrm{mg}$ doses producing the most significant improvement. When individual fexofenadine groups were compared with placebo, only the $180 \mathrm{mg}$ fexofenadine showed significant reductions in the MNW with a decrease of $0.52 \pm 0.19$.

Acrivastine.

Gibson et al.[24] reported that acrivastine at $4 \mathrm{mg}$ and $8 \mathrm{mg}$ significantly reduced symptoms of urticaria compared with placebo. Acrivastine has a rapid therapeutic effect, which reached its peak within two hours.

Desloratadine.

Weller et al.[25] reported that $5 \mathrm{mg}$ and $20 \mathrm{mg}$ on-demand treatment of desloratadine led to an effective reduction in the hyperthermic skin area, but there was no improvement in wheal area, pruritus, and global efficacy compared with no treatment.

\section{Bilastine}

Hide et al.[26] reported that Bilastine at 10 and 20 mg administered over a two-week period resulted in a decrease in TSS from baseline of 3.3 and 3.01 respectively, which were significantly better than placebo (with a 1.49 reduction). Bilastine at $10 \mathrm{mg}$ and 20 mg significantly improved wheal and pruritus when compared with placebo. The effectiveness of bilastine once daily could last throughout the day.

Cetirizine. 
Kalivas et al.[31] reported on $69 \mathrm{CSU}$ patients treated with cetirizine once daily for four weeks at a dose of approximately 5 to $20 \mathrm{mg}$. Cetirizine was better than the placebo at reducing the number and size of lesions, the number of urticarial attacks, and the severity of pruritus.

Combined use of sgAHs.

Staevska et al.[30] studied two groups of CSU patients receiving $5 \mathrm{mg}$ of either desloratadine or levocetirizine in the first week. If this dose was not successful within the next week, the dose was doubled during the following week up to a maximum of four times of the standard dose. The two groups switched the two types of treatment up to a maximum of four times the standard dose of sgAHs in the fourth week. There were significant differences in the number of successful treatments comparing high and standard doses for both levocetirizine and desloratadine. The overall success rate of 22 patients with levocetirizine was significantly higher than the rate of the 12 patients treated with desloratadine at the end of week 3 . At the end of the third week, patients who were still symptomatic switched to the opposite drug. Seven patients who did not respond to $20 \mathrm{mg}$ of desloratadine had no more symptoms after taking $20 \mathrm{mg}$ of levocetirizine, while there was no benefit in switching to loratadine in 18 patients who had not been cured with 20 mg of levocetirizine.

Sánchez et al.[29] reported on 150 CSU patients (30 per group) receiving a daily oral standard dose of ebastine (20 mg), bilastine (20 mg), fexofenadine $(180 \mathrm{mg})$, cetirizine $(10 \mathrm{mg})$ or desloratadine $(5 \mathrm{mg})$ over four weeks, respectively. After four weeks, the sgAHs dose was modified up to approximately two or four times the standard dose according to its clinical effectiveness and adverse reactions. There was no significant difference in disease control among the groups. After four weeks of antihistamine treatment using standard doses, the symptoms were completely controlled in $58.7 \%$ of patients ( $\mathrm{n}=88$ ) and partially controlled in $30.7 \%(n=46)$ of patients. Clinical response in patients with DLQI greater than 5 improved in most patients when the antihistamine dose was increased, with $76.7 \%(n=115)$ of patients having their symptoms fully controlled, $15.3 \%$ partially controlled $(n=23)$, and $6.7 \%$ uncontrolled $(n=10)$.

\section{Adverse Events}

A total of six studies[21-23, 27, 28, 32] reported adverse events while no deaths occurred. Four serious adverse events[22, 23, 32] were reported, but they were not significantly associated with sgAHs treatment. There was no significant difference in the incidence of adverse events between high dose and standard dose (RR 1.05, 95\% Cl 0.92 to $1.21 ; \mathrm{P}=0.44)$ in sgAHs treatment. Somnolence was the most concerning adverse event experienced in high-dose groups, which was reported by four studies[21, 22, 27, 32]. Higher doses of sgAHs were associated with a higher incidence of somnolence when compared with standard dose (RD $0.05,95 \% \mathrm{Cl} 0.01$ to $0.09 ; \mathrm{P}=0.02)$. Headache $(16.2 \%, \mathrm{n}=84)$ was the most common adverse event experience in high-dose groups, followed by upper respiratory infection $(10.9 \%, n=37)$, somnolence $(9.0 \%, n=32)$, nasopharyngitis $(7.1 \%, n=32)$ and gastrointestinal symptoms $(8.2 \%, n=28)$. Five studies $[21,23,27,28,32]$ reported on the need to withdraw treatment due to adverse events. Compared with the standard doses, a high-dose treatment did not increase the need to withdraw treatment due to adverse events $(n=725, R R 0.63,95 \% \mathrm{Cl} 0.29$ to $1.36 ; P=0.24)$ except for fexofenadine. Fexofenadine was the drug with the most reported adverse events in the high-dose group, but rarely including somnolence.

\section{Discussion}

Treatment with sgAHs is the preferred management of CSU as it is safe, convenient, and cost-effective. Both European[1] and American guidelines[10] recommend increasing the dose of sgAHs as a second-line treatment for CSU. However, studies on the efficacy and safety of using a high dose of sgAHs for the treatment of CSU are limited and still inconclusive[33, 34]. European[1], British[35], American guidelines[10], Chinese[36] and Japanese[37] guidelines recommend increasing the dose of sgAHs up two to four times the recommended dose. Higher doses of sgAHs might provide more efficacy, but current data are limited and conflicting for certain agents[10]. Both the European and American guidelines recommend using the lowest number and safest medications to manage CSU[38]. The premise of increasing sgAHs dose is that high dose of sgAHs is more effective than the standard-dose sgAHs. If high-dose sgAHs cannot improve the efficacy, increasing the dose of sgAHs is of little significance, and alternative treatment options should be considered as soon as possible. On the other hand, if the high dose proves to be beneficial, it will provide strong evidence for the development of new consensus guidelines.

We, therefore, performed a meta-analysis on the treatment of CSU with high-dose sgAHs to clarify the issue with a special focus on safety, since a previous meta-analysis conducted by Guillén-Aguinaga et al.[12] only reported on efficacy. The findings of our meta-analysis suggest that high-dose treatment up to a maximum of double the standard dose of sgAHs might provide a better response rate when compared with conventional treatment in patients suffering from CSU. High-dose and standard-dose sgAHs showed similar safety profiles. However, this improvement came at the cost of increasing specific adverse events, with somnolence being reported as most distressing for the patient. The overall result of our meta-analysis identified the prevalence of somnolence as being dose-dependent. However, this result seemed to be heavily influenced by one of the Dubertret L's study[32], whereby its exclusion ultimately resulted in no difference in the somnolence incidence between high dose and standard dose of sgAHs.

In our meta-analysis, the response rate using the standard dose was $64.5 \%$ and $71.2 \%$ in the high-dose treatment. Our results are inconsistent with GuillénAguinaga's[12], which may be due to different defining criteria for response rates. Guillén-Aguinaga defined the failed treatment response as an overall symptom improvement of less than $50 \%$ or treatment termination due to failure, while we defined the respondent patients according to the overall degree of improvement as indicated in the original text. We are concerned about whether high-dose sgAHs can improve the efficacy of CSU.

There are a number of factors that may lead to poor response following high-dose treatment. CSU is a self-limiting disease, and urticarial activity tends to relapse over time[12,39]. This implies that the therapeutic effect may be dose- and time-dependent[25, 32], and therefore, continuous and regular medication might provide more effective symptom relief in CSU patients. Furthermore, the findings of the three studies included in our meta-analysis revealed no significant difference between low and standard sgAHs doses ( $n=415, \mathrm{RR} 1.09,95 \% \mathrm{Cl} 0.96$ to $1.25 ; \mathrm{P}=0.18)$. The effect of sgAHs on response rate may not necessarily be linked with the dose, and therefore, further high-quality studies evaluating the impact of dose and time response are needed. 
Although treatment with sgAHs may be accompanied by headache, somnolence, nasopharyngitis, and other side effects, these adverse events are reported to be rare, mild, and transient in both high- and standard-dose groups. Somnolence is an adverse event of major concern for both patients and doctors,

eventually limiting dose escalation of sgAHs[40]. A total of four studies evaluated the incidence of somnolence, with a total of 63 cases of somnolence (6.3\%) being reported after taking sgAHs. The incidence rate was higher in the high-dose group (9\%) when compared with standard treatment (5\%).

The strong point of our meta-analysis was that we performed the first safety and adverse events evaluation of using high-dose sgAHs for the treatment of CSU. However, our meta-analysis has some limitations that have to be acknowledged, and therefore, our results should be interpreted with caution. First of all, we did not evaluate all sgAHs. Since different sgAHs are known to exhibit different pharmacokinetic and pharmacodynamic properties, it cannot be ruled out that the use of other antihistamines may lead to different results. The number of studies evaluated in this meta-analysis was small, limiting the generalizability of the research findings. Studies comparing efficacy and safety between standard-and high-dose sgAHs only escalated the dose up to twice the standard dose but not up to four times as recommended by the European guidelines[1]. Moreover, there was considerable variability in the indices used to measure treatment outcomes, and not all outcomes were reported in each study. Therefore, we did not have sufficient data to evaluate the improvement of pruritus, wheals, and DLQI.

\section{Conclusion}

The findings of the meta-analyses showed that high-dose sgAHs (up to two times the standard dose) might be more effective than standard doses in the treatment of CSU. High-dose and standard-dose sgAHs showed similar safety profiles, with the exception of somnolence that might be dose-dependent. However, due to the limited number of studies in our meta-analysis, results should be interpreted with caution.

\section{Abbreviations}

CSU, chronic spontaneous urticaria; sgAHs, second-generation $\mathrm{H}_{1}$-antihistamines; RCT, randomized controlled trials; DLQI, Dermatology Life Quality Index; VAS, visual analogue scale; MPS, mean pruritus score; MNW, mean number of wheals; MTSS, calculated as the sum of MPS and MNW;

\section{Declarations}

\section{Acknowledgments}

We would like to thank TopEdit (www.topeditsci.com) for the English language editing of this manuscript.

\section{Authors' Contributions:}

XJX was responsible for Conceptualization. YZS, LXZ, RJJ and WC were responsible for data curation. RJJ, XJX and SYZ were responsible for formal analysis. WC, ZHZ and CW were responsible for investigation. XJX, JPY and YZS were responsible for methodology. XJX and YZS were responsible for project administration. YL and QHZ were responsible for supervision. XJX was responsible for original draft. MLC was responsible for review and editing.

\section{Funding}

This study was supported by the National Key R\&D Program of China "Research on Modernization of Traditional Chinese Medicine"-"International Cooperation Research on Evaluation of Acupuncture Advantage Diseases" (grant numbers 2017 YFC1703600 and 2017YFC1703605).

\section{Availability of data and materials}

All data generated or analysed during this study are included in this published article.

\section{Ethics approval and consent to participate:}

Not Applicable.

\section{Consent for publication:}

Not Applicable.

\section{Competing interests:}

The authors declare that they have no competing interests.

\section{References}

1. Zuberbier T, Aberer W, Asero R, Abdul Latiff AH, Baker D, Ballmer-Weber B et al. The EAACI/GA(2)LEN/EDF/WAO guideline for the definition, classification, diagnosis and management of urticaria. Allergy. 2018;73(7):1393-414.

2. Fricke J, Avila G, Keller T, Weller K, Lau S, Maurer M et al. Prevalence of chronic urticaria in children and adults across the globe: Systematic review with meta-analysis. Allergy. 2020;75(2):423-32. 
3. Maurer M, Weller K, Bindslev-Jensen C, Gimenez-Arnau A, Bousquet PJ, Bousquet J et al. Unmet clinical needs in chronic spontaneous urticaria. A GA(2)LEN task force report. Allergy. 2011;66(3):317-30.

4. Goldstein S, Eftekhari S, Mitchell L, Winders TA, Kaufman L, Dudas D et al. Perspectives on Living with Chronic Spontaneous Urticaria: From Onset through Diagnosis and Disease Management in the US. Acta Derm Venereol. 2019;99(12):1091-8.

5. Ben-Shoshan M, Blinderman I, Raz A. Psychosocial factors and chronic spontaneous urticaria: a systematic review. Allergy. 2013;68(2):131-41.

6. Tzur Bitan D, Berzin D, Cohen A. The association of chronic spontaneous urticaria (CSU) with anxiety and depression: a nationwide cohort study. Arch Dermatol Res. 2020.

7. Ferrer M. Epidemiology, healthcare, resources, use and clinical features of different types of urticaria. Alergologica 2005. J Investig Allergol Clin Immunol. 2009;19 Suppl 2:21-6.

8. Weller K, Viehmann K, Brautigam M, Krause K, Siebenhaar F, Zuberbier T et al. Cost-intensive, time-consuming, problematical? How physicians in private practice experience the care of urticaria patients. J Dtsch Dermatol Ges. 2012;10(5):341-7.

9. Carrillo-Martin I, Dudgeon MG, Chamorro-Pareja N, Haehn DA, Rivera-Valenzuela MG, Spaulding AC et al. Cost-Utility of Routine Testing in Chronic Urticaria/Angioedema: A Cohort Study. J Allergy Clin Immunol Pract. 2019;7(8):2823-32.

10. Bernstein JA, Lang DM, Khan DA, Craig T, Dreyfus D, Hsieh F et al. The diagnosis and management of acute and chronic urticaria: 2014 update. J Allergy Clin Immunol. 2014;133(5):1270-7.

11. Weller K, Maurer M. Antihistamine updosing in chronic urticaria - is there enough evidence? Br J Dermatol. 2016;175(6):1134-5.

12. Guillen-Aguinaga S, Jauregui Presa I, Aguinaga-Ontoso E, Guillen-Grima F, Ferrer M. Updosing nonsedating antihistamines in patients with chronic spontaneous urticaria: a systematic review and meta-analysis. Br J Dermatol. 2016;175(6):1153-65.

13. Vestergaard C, Toubi E, Maurer M, Triggiani M, Ballmer-Weber B, Marsland A et al. Treatment of chronic spontaneous urticaria with an inadequate response to H1-antihistamines: an expert opinion. Eur J Dermatol. 2017;27(1):10-9.

14. Manti S, Salpietro C, Cuppari C. Antihistamines: Recommended Dosage - Divergence between Clinical Practice and Guideline Recommendations. International archives of allergy and immunology. 2019;178(1):93-6.

15. Moher D, Shamseer L, Clarke M, Ghersi D, Liberati A, Petticrew M et al. Preferred reporting items for systematic review and meta-analysis protocols (PRISMA-P) 2015 statement. Syst Rev. 2015;4:1.

16. Furlan AD, Pennick V, Bombardier C, van Tulder M, Editorial Board CBRG. 2009 updated method guidelines for systematic reviews in the Cochrane Back Review Group. Spine (Phila Pa 1976). 2009;34(18):1929-41.

17. Gheibi S, Mahmoodzadeh A, Kashfi K, Jeddi S, Ghasemi A. Data Extraction from Graphs Using Adobe Photoshop: Applications for Meta-Analyses. Int J Endocrinol Metab. 2019;17(4):e95216.

18. Karri J, Orhurhu V, Wahezi S, Tang T, Deer T, Abd-Elsayed A. Comparison of Spinal Cord Stimulation Waveforms for Treating Chronic Low Back Pain: Systematic Review and Meta-Analysis. Pain Physician. 2020;23(5):451-60.

19. Mazaud C, Fardet L. Relative risk of and determinants for adverse events of methotrexate prescribed at a low dose: a systematic review and metaanalysis of randomized placebo-controlled trials. Br J Dermatol. 2017;177(4):978-86.

20. Rong LQ, Kamel MK, Rahouma M, Naik A, Mehta K, Abouarab AA et al. High-dose versus low-dose opioid anesthesia in adult cardiac surgery: A metaanalysis. J Clin Anesth. 2019;57:57-62.

21. Paul E, Berth-Jones J, Ortonne JP, Stern M. Fexofenadine hydrochloride in the treatment of chronic idiopathic urticaria: A placebo-controlled, parallelgroup, dose-ranging study. Journal of Dermatological Treatment. 1998;9(3):143-9.

22. Gimenez-Arnau A, Pujol RM, lanosi S, Kaszuba A, Malbran A, Poop G et al. Rupatadine in the treatment of chronic idiopathic urticaria: a double-blind, randomized, placebo-controlled multicentre study. Allergy. 2007;62(5):539-46.

23. Finn AF, Jr., Kaplan AP, Fretwell R, Qu R, Long J. A double-blind, placebo-controlled trial of fexofenadine $\mathrm{HCl}$ in the treatment of chronic idiopathic urticaria. J Allergy Clin Immunol. 1999;104(5):1071-8.

24. Gibson JR, Harvey SG, Barth JH, Moss MY, Burke CA. An assessment of the novel antihistamine BW $825 \mathrm{C}$ in the treatment of chronic idiopathic urticaria. A placebo-controlled study. Dermatologica. 1984;169(4):179-83.

25. Weller K, Ardelean E, Scholz E, Martus P, Zuberbier T, Maurer M. Can on-demand non-sedating antihistamines improve urticaria symptoms? A double-blind, randomized, single-dose study. Acta Derm Venereol. 2013;93(2):168-74.

26. Hide M, Yagami A, Togawa M, Saito A, Furue M. Efficacy and safety of bilastine in Japanese patients with chronic spontaneous urticaria: A multicenter, randomized, double-blind, placebo-controlled, parallel-group phase II/III study. Allergol Int. 2017;66(2):317-25.

27. Hide M, Suzuki T, Tanaka A, Aoki H. Efficacy and safety of rupatadine in Japanese adult and adolescent patients with chronic spontaneous urticaria: A double-blind, randomized, multicenter, placebo-controlled clinical trial. Allergol Int. 2019;68(1):59-67.

28. Nelson HS, Reynolds R, Mason J. Fexofenadine $\mathrm{HCl}$ is safe and effective for treatment of chronic idiopathic urticaria. Ann Allergy Asthma Immunol. 2000;84(5):517-22.

29. Sanchez J, Zakzuk J, Cardona R. Prediction of the Efficacy of Antihistamines in Chronic Spontaneous Urticaria Based on Initial Suppression of the Histamine-Induced Wheal. J Investig Allergol Clin Immunol. 2016;26(3):177-84.

30. Staevska M, Popov TA, Kralimarkova T, Lazarova C, Kraeva S, Popova D et al. The effectiveness of levocetirizine and desloratadine in up to 4 times conventional doses in difficult-to-treat urticaria. J Allergy Clin Immunol. 2010;125(3):676-82. 
31. Kalivas J, Breneman D, Tharp M, Bruce S, Bigby M. Urticaria: clinical efficacy of cetirizine in comparison with hydroxyzine and placebo. J Allergy Clin Immunol. 1990;86(6 Pt 2):1014-8.

32. Dubertret L, Zalupca L, Cristodoulo T, Benea V, Medina I, Fantin S et al. Once-daily rupatadine improves the symptoms of chronic idiopathic urticaria: a randomised, double-blind, placebo-controlled study. Eur J Dermatol. 2007;17(3):223-8.

33. Asero R. Chronic unremitting urticaria: is the use of antihistamines above the licensed dose effective? A preliminary study of cetirizine at licensed and above-licensed doses. Clin Exp Dermatol. 2007;32(1):34-8.

34. Okubo Y, Shigoka Y, Yamazaki M, Tsuboi R. Double dose of cetirizine hydrochloride is effective for patients with urticaria resistant: a prospective, randomized, non-blinded, comparative clinical study and assessment of quality of life. J Dermatolog Treat. 2013;24(2):153-60.

35. Powell RJ, Leech SC, Till S, Huber PA, Nasser SM, Clark AT et al. BSACl guideline for the management of chronic urticaria and angioedema. Clin Exp Allergy. 2015;45(3):547-65.

36. Xu J. Guideline for diagnosis and treatment of urticaria in China. Chin J Dermatol. 2019;52(1):1-5.

37. Hide M. [Japanese Guidelines for Diagnosis and Treatment of Urticaria 2018]. Arerugi. 2018;67(10):1394-8.

38. Zuberbier T, Bernstein JA. A Comparison of the United States and International Perspective on Chronic Urticaria Guidelines. J Allergy Clin Immunol Pract. 2018;6(4):1144-51.

39. Kameyoshi Y, Tanaka T, Mihara S, Takahagi S, Niimi N, Hide M. Increasing the dose of cetirizine may lead to better control of chronic idiopathic urticaria: an open study of 21 patients. Br J Dermatol. 2007;157(4):803-4.

40. Weller K, Ziege C, Staubach P, Brockow K, Siebenhaar F, Krause K et al. H1-antihistamine up-dosing in chronic spontaneous urticaria: patients' perspective of effectiveness and side effects-a retrospective survey study. PLoS One. 2011;6(9):e23931.

\section{Tables}


Table.1: Characteristics of studies included in the meta-analysis

\begin{tabular}{|c|c|c|c|c|c|c|c|c|c|c|}
\hline Study & $\begin{array}{l}\text { Sample } \\
\text { Size } \\
\text { (female,\%) }\end{array}$ & Age & $\begin{array}{l}\text { Course } \\
\text { of the } \\
\text { disease } \\
\text { (week) }\end{array}$ & $\begin{array}{l}\text { Outcome } \\
\text { measurement }\end{array}$ & Intervention & TD & $\begin{array}{l}\text { Definition of } \\
\text { response }\end{array}$ & $\begin{array}{l}\text { Response } \\
\text { rate*, } \\
\text { no. (\%) }\end{array}$ & $\begin{array}{l}\text { Adverse } \\
\text { events, } \\
\text { no. (\%) }\end{array}$ & $\begin{array}{l}\text { Somnolence } \\
\text { (\%) }\end{array}$ \\
\hline \multirow{5}{*}{$\begin{array}{l}\text { Gimenez- } \\
\text { Arnau et } \\
\text { al. } \\
(2007)\end{array}$} & \multirow[t]{5}{*}{$\begin{array}{l}329 \\
(68.39 \%)\end{array}$} & \multirow[t]{5}{*}{$\begin{array}{l}12- \\
65\end{array}$} & \multirow[t]{5}{*}{$\geq 6$} & \multirow{2}{*}{$\begin{array}{l}\text { 5-point rating } \\
\text { score } \mathbb{Q} \text { (MPS, } \\
\text { MNW, MTSS) }\end{array}$} & $\begin{array}{l}\text { Rupatadine } \\
10 \mathrm{mg}\end{array}$ & \multirow[t]{5}{*}{$\begin{array}{l}6 \\
\text { weeks }\end{array}$} & \multirow{5}{*}{$\begin{array}{l}\text { pruritus } \\
\text { symptoms } \\
\text { reduction } \\
\text { higher than } \\
50 \%\end{array}$} & & \multirow{3}{*}{$\begin{array}{l}13 \\
(11.6 \%) \\
18 \\
(16.5 \%)\end{array}$} & $3(2.7 \%)$ \\
\hline & & & & & & & & 79 (73.15\%) & & $9(8.3 \%)$ \\
\hline & & & & \multirow{2}{*}{$\begin{array}{l}\text { 5-point rating } \\
\text { score \& } \\
\text { (Global } \\
\text { efficacy); }\end{array}$} & $20 \mathrm{mg}$ & & & $51(45.9 \%)$ & & $6(5.3 \%)$ \\
\hline & & & & & Placebo & & & & $\begin{array}{l}13 \\
(11.5 \%)\end{array}$ & \\
\hline & & & & DLQI & & & & & & \\
\hline
\end{tabular}

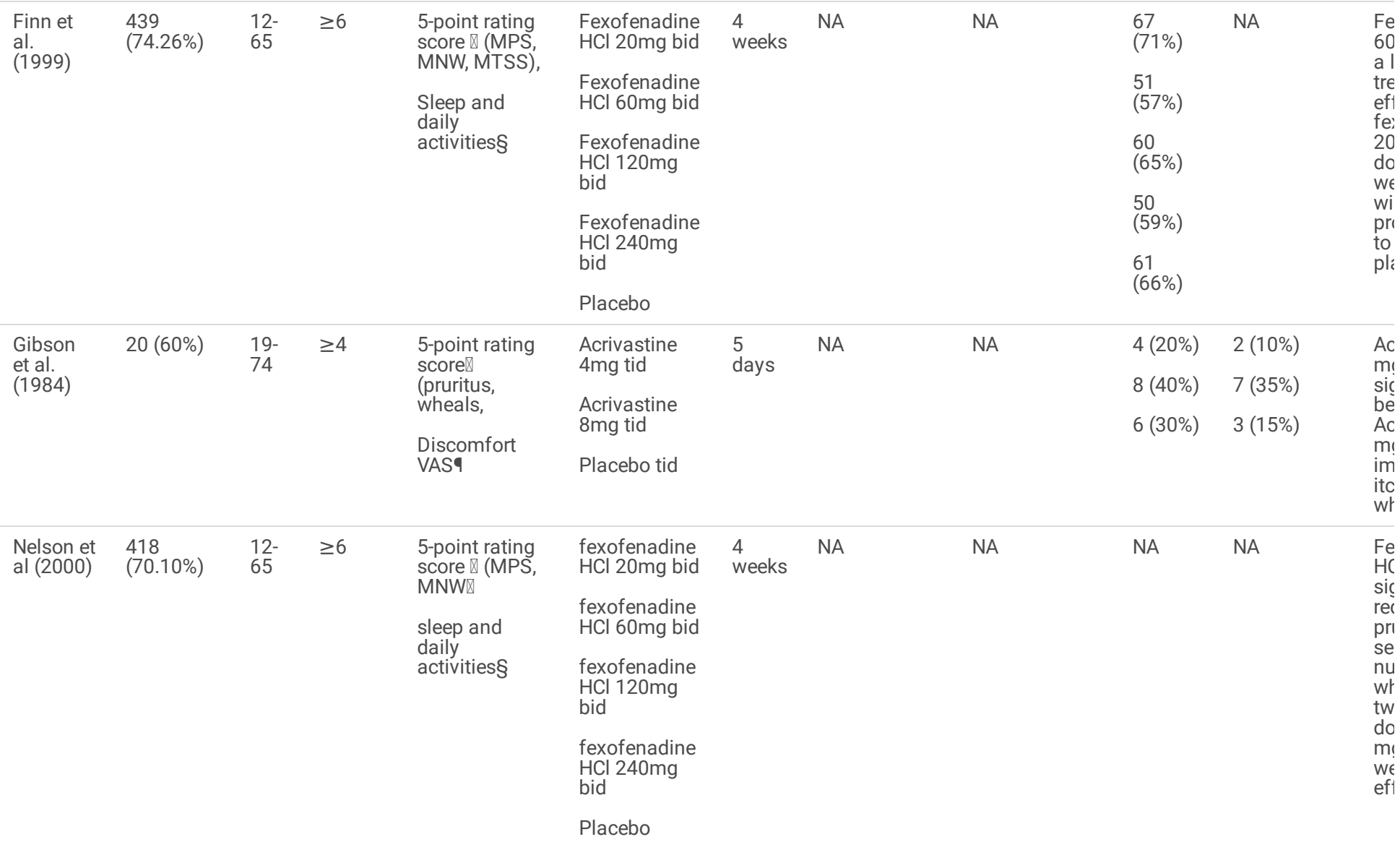

\begin{tabular}{|c|c|c|c|c|c|c|c|c|c|c|}
\hline \multirow[t]{7}{*}{$\begin{array}{l}\text { Paul et al } \\
\text { (1998) }\end{array}$} & \multirow[t]{7}{*}{$\begin{array}{l}208 \\
(57.69 \%)\end{array}$} & \multirow[t]{7}{*}{$\geq 18$} & \multirow[t]{7}{*}{$\geq 6$} & \multirow{3}{*}{ 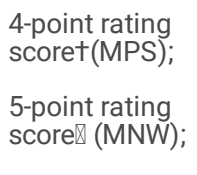 } & \multirow[t]{2}{*}{$\begin{array}{l}\text { Fexofenadine } \\
\mathrm{HCl} 60 \mathrm{mg}\end{array}$} & \multirow[t]{7}{*}{$\begin{array}{l}6 \\
\text { weeks }\end{array}$} & \multirow{7}{*}{$\begin{array}{l}\text { rated the } \\
\text { effectiveness } \\
\text { of the } \\
\text { medication } \\
\text { as good, very } \\
\text { good or } \\
\text { excellent }\end{array}$} & $25(63 \%)$ & $7(18 \%)$ & $0(0 \%)$ \\
\hline & & & & & & & & 18 (50\%) & $9(26 \%)$ & $0(0 \%)$ \\
\hline & & & & & $\mathrm{HCl} 120 \mathrm{mg}$ & & & 30 (64\%) & 13 & $0(0 \%)$ \\
\hline & & & & TSS; & Fexofenadine & & & $21(55 \%)$ & $(200$ & $0(0 \%)$ \\
\hline & & & & Sleep and & HCl 180mg & & & $19(41 \%)$ & $\begin{array}{l}10 \\
(26 \%)\end{array}$ & $0(0 \%)$ \\
\hline & & & & activities§; & $\mathrm{HCl} 240 \mathrm{mg}$ & & & & $\begin{array}{l}15 \\
(33 \%)\end{array}$ & \\
\hline & & & & $\begin{array}{l}\text { Medication } \\
\text { effectiveness } \emptyset\end{array}$ & Placebo & & & & & \\
\hline
\end{tabular}

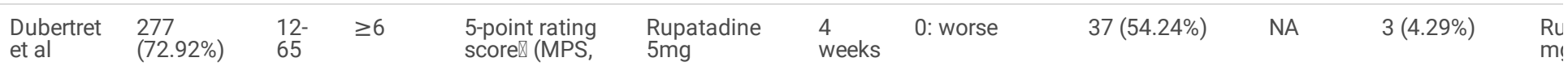




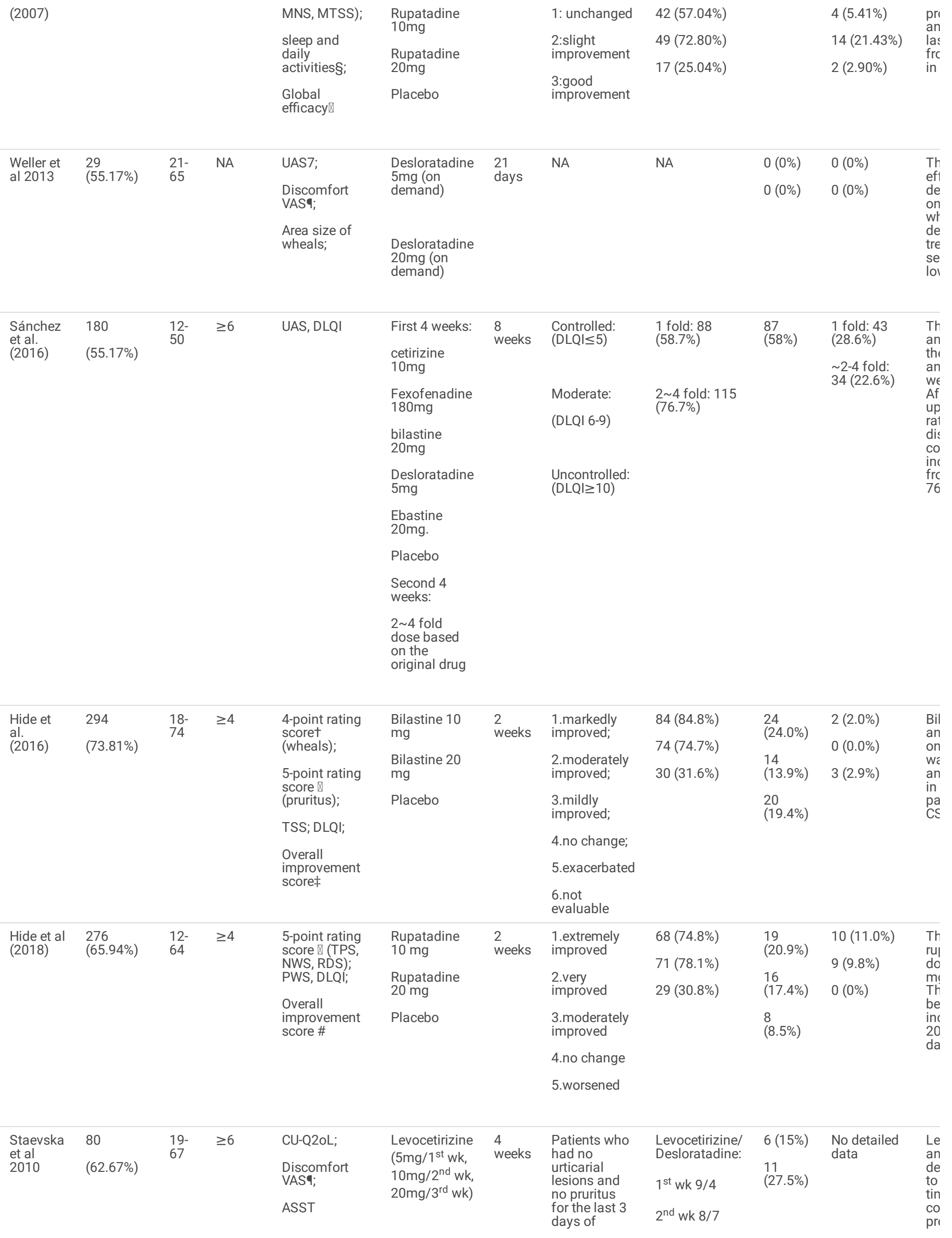




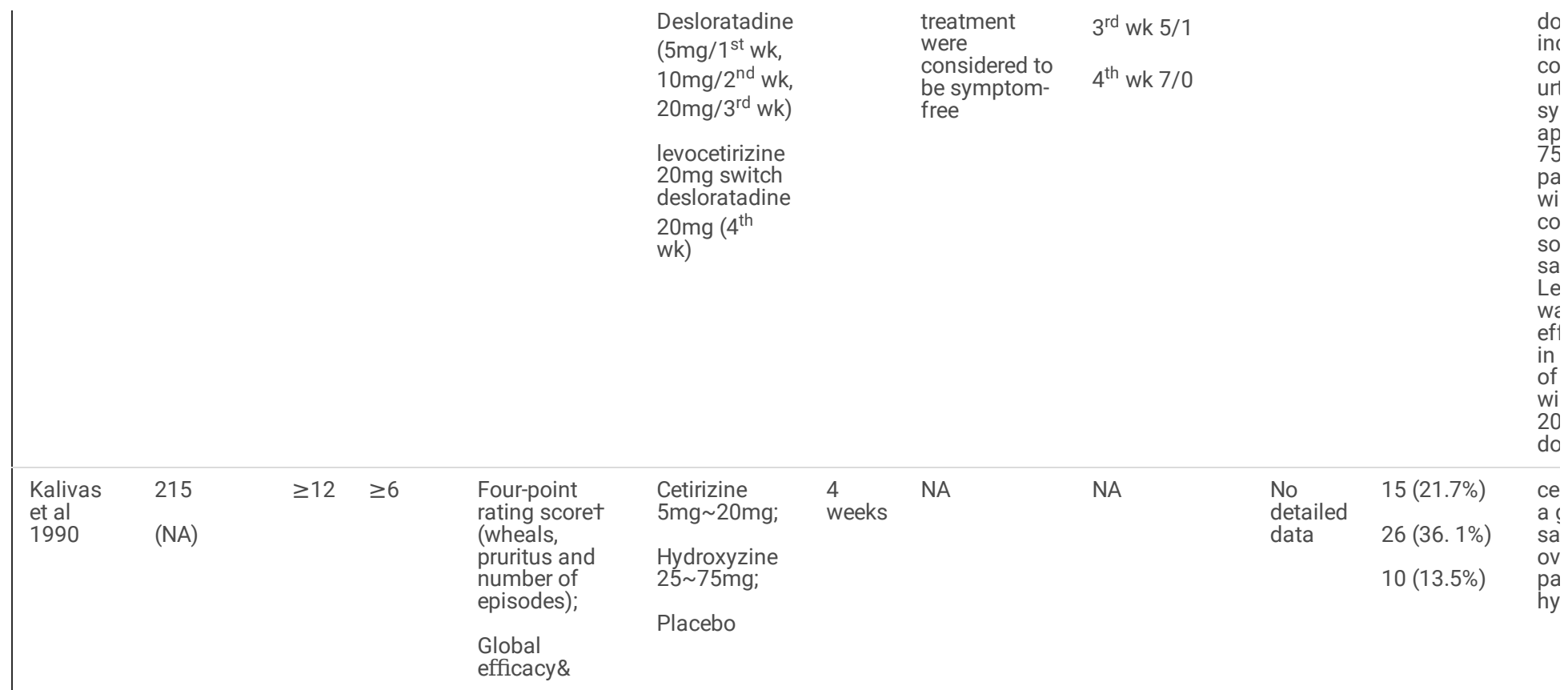

Abbreviations: TD: Treatment duration, DLQI, Dermatology Life Quality Index; VAS, visual analogue scale; MPS, mean pruritus score; MNW, mean number of n MTSS, calculated as the sum of MPS and MNW; NA, data were not available in the study; TSS, the sum of the wheal and pruritus scores; TPS, total pruritus S number of wheals score; RDS, rash duration score; PWS, the sum of pruritus and number of wheals score; UAS, Urticaria Activity Score; ASST: Autologous ser CU-Q2oL: Chronic urticaria quality of life questionnaire.

*Response, pruritus symptoms reduction higher than $50 \%$, or overall improvement rated at least moderately/very improved

$\triangle$ Five-point rating score (0-4): $0=$ no symptom to $4=$ the worst symptom, the higher the score, the worse the symptoms;

\& Global efficacy (0-4): 0 = worse to $4=$ excellent improvement, the higher the score, the better the symptoms;

$\S$ sleep and daily activities: (0-3): $0=$ none, $1=$ mild, $2=$ moderate, and $3=$ severe.

१Discomfort VAS: patients evaluated their drowsiness, itching and severity of symptoms by marking along a 0-100 mm long horizontal line $(0=$ min to 106 =

tFour-point rating score (0-3): $0=$ no symptom to $3=$ the worst symptom, the higher the score, the worse the symptoms;

‡ Five-point rating score (1-5): 1 = markedly improved to 5 = exacerbated, the higher the score, the worse the symptoms;

\# Overall improvement score (1-6): 1 = extremely improved, $2=$ very improved, $3=$ moderately improved, $4=$ no change, $5=$ worsened, and $6=$ not evaluable;

$\emptyset$ medication effectiveness $(0-4,0=$ excellent to $4=$ none $)$

\section{Figures}




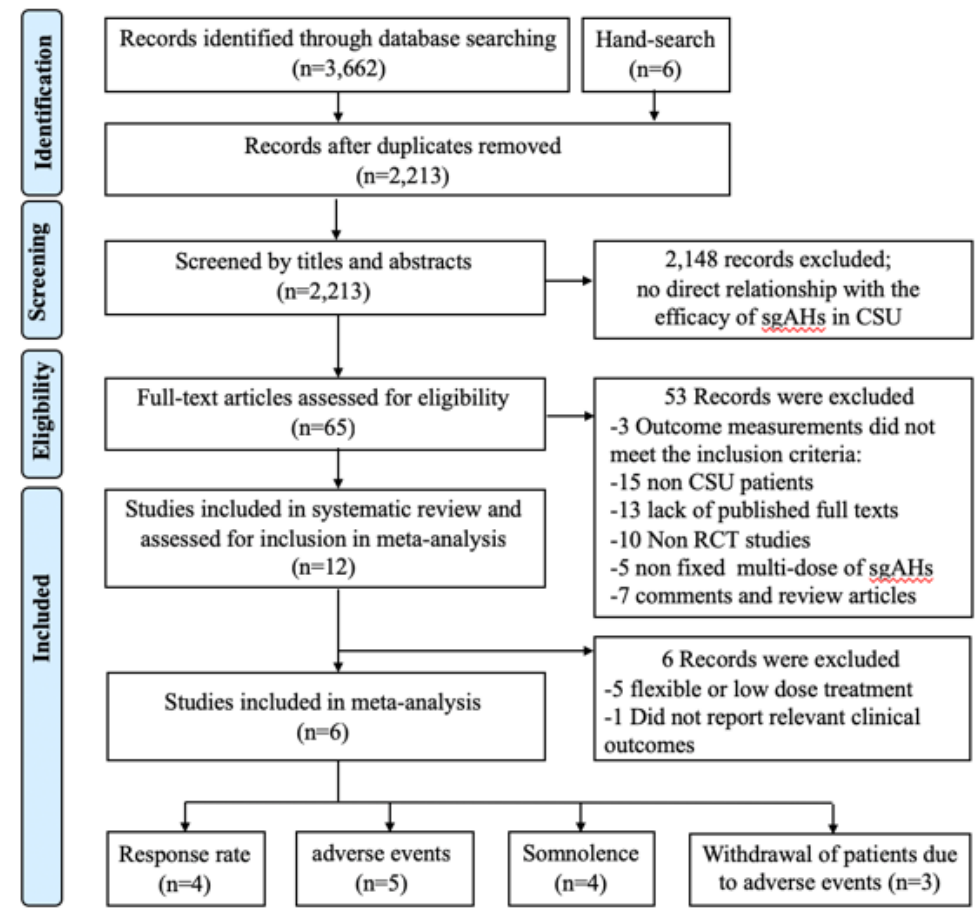

\section{Figure 1}

Flow diagram illustrating the search strategy used to identify suitable studies.

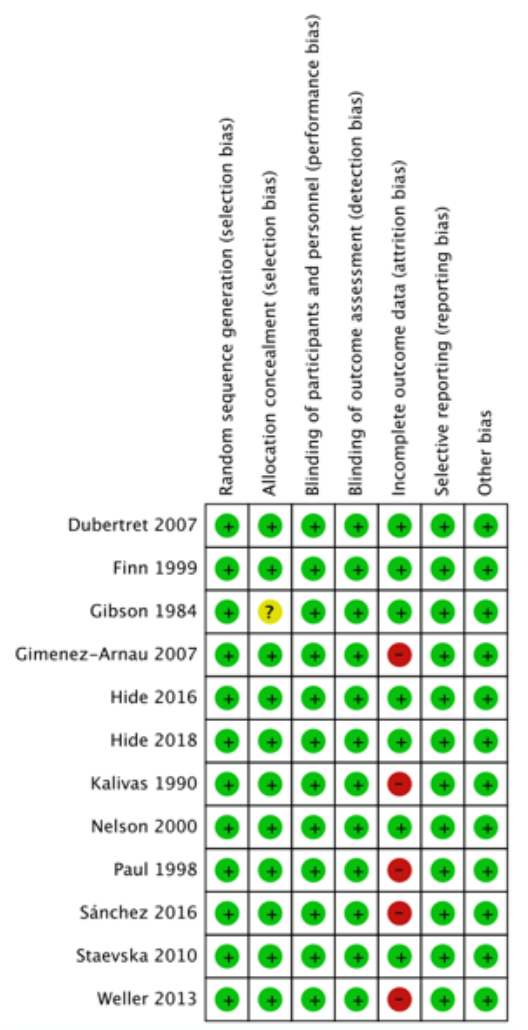

Figure 2

Risk of bias of the included studies. 


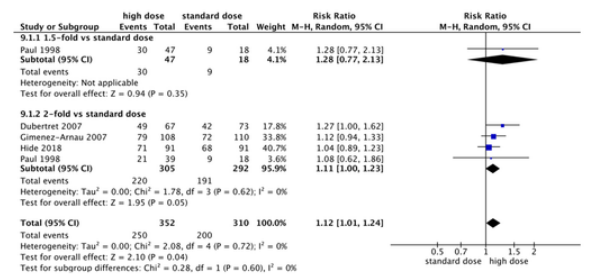

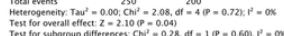

(A)

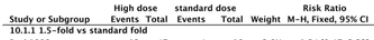

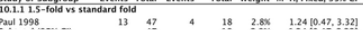

\section{Figure 3}

A: Forest plot of response rate comparing high-dose with standard-dose sgAHs.

B: Forest plot of adverse events comparing high-dose with standard-dose sgAHs.

C: Forest plot of somnolence comparing high-dose with standard-dose sgAHs.

D: Forest plot of comparing withdrawal of patients due to adverse events between high-dose and standard-dose sgAHs treatment

\section{Supplementary Files}

This is a list of supplementary files associated with this preprint. Click to download.

- PRISMA2020checklist.docx 